\title{
Mild solutions to fractional differential inclusions with nonlocal conditions
}

\author{
Tingting Lian' ${ }^{1}$ Changfeng $X \mathrm{Xe}^{1}$ and Shaozhong Deng ${ }^{2 *}$ (D)
}

"Correspondence:
shaodeng@uncc.edu
${ }^{2}$ Department of Mathematics and
Statistics, University of North
Carolina at Charlotte, Charlotte, NC
28223, United States
Full list of author information is
available at the end of the article

available at the end of the article

\begin{abstract}
This article is concerned with the existence of mild solutions for fractional differential inclusions with nonlocal conditions in Banach spaces. The results are obtained by using fractional calculus, Hausdorff measure of noncompactness, and the multivalued fixed point theorem. The results obtained in the present paper extend some related results on this topic.
\end{abstract}

Keywords: fractional differential inclusions; nonlocal conditions; Hausdorff measure of noncompactness; equicontinuous semigroup; mild solution

\section{Introduction}

Fractional differential equations and inclusions have gained considerable interest due to their applications in various fields, such as physics, mechanics, and engineering, in part because they have been found to be more realistic and practical to describe many natural phenomena [1-8]. For more details about fractional calculus and fractional differential equations, we refer the reader to the books by Podlubny [9], Sabatier et al. [10], Kilbas et al. [11], and the papers by Eidelman and Kochubei [12], Lakshmikantham and Vatsala [13], and Agarwal et al. [14].

The study of abstract nonlocal differential problems was initiated by Byszewski and Lakshmikantham [15], who gave three theorems on the existence and uniqueness of the mild, strong, and classical solutions of a semilinear evolution nonlocal Cauchy problem by using the method of semigroups and the Banach fixed point theorem and argued there that a nonlocal condition can be applied in physics with better effects than classical initial conditions. This work was then followed by a lot of other research, and some basic results on nonlocal differential problems have been obtained [16-18]. Most results, however, were obtained with the assumption that the involved semigroup is compact, and one of the difficulties in these nonlocal problems is how to deal with the compactness of the solution operator at $t=0$. Many methods and techniques have been developed to avoid this difficulty. We refer the reader to papers [19-21] and the references therein.

From the mathematical point of view, it is natural to combine fractional differential equations with nonlocal conditions. For example, Zhou and Jiao [22] discussed the nonlocal fractional evolution equations based on the Krasnoselskii fixed point theorem with the assumption that the involved semigroup is compact and the nonlocal term is Lipschitz continuous. Li et al. [23] studied the existence of mild solutions to fractional differential equations by using the Hausdorff measure of noncompactness when the semigroup

(c) The Author(s) 2016. This article is distributed under the terms of the Creative Commons Attribution 4.0 International License (http://creativecommons.org/licenses/by/4.0/), which permits unrestricted use, distribution, and reproduction in any medium, provided you give appropriate credit to the original author(s) and the source, provide a link to the Creative Commons license, and indicate if changes were made. 
is equicontinuous and the nonlocal term is compact. Ji and $\mathrm{Li}$ [24] also studied nonlocal fractional differential equations in general Banach spaces but without any compactness assumptions to the operator semigroup. Recently, Ji [25] studied the control system governed by a class of abstract nonlocal fractional differential equations. To the best of the authors' knowledge, however, few work has been reported on the existence of solutions for fractional differential inclusions with nonlocal conditions governed by a linear closed operator that generates an equicontinuous semigroup.

In this paper, we assume that $X$ is a real Banach space with norm $\|\cdot\|$. Let $J=[0, b]$ with $b>0$. We denote by $C(J, X)$ the Banach space of continuous functions from $J$ into $X$ with the norm $\|x\|=\sup _{t \in J}\{\|x(t)\|\}$ for $x \in C(J, X)$. We further denote by $L^{1}(J, X)$ the space of Bochner-integrable functions from $J$ into $X$ with the norm given by $\|f\|_{L^{1}}=\int_{0}^{b}\|f(t)\| \mathrm{d} t$ for $f \in L^{1}(J, X)$.

Motivated by the aforementioned previous research, we study the existence of mild solutions to the following fractional semilinear nonlocal differential inclusion:

$$
\begin{aligned}
& { }^{C} D_{t}^{q} u(t) \in A u(t)+F(t, u(t)), \quad t \in J, \\
& u(0)=g(u),
\end{aligned}
$$

where $0<q \leq 1,{ }^{C} D_{t}^{q}$ is the Caputo fractional differential operator of order $q, u \in C(J, X)$, $A: D(A) \rightarrow X$ is the infinitesimal generator of a strongly continuous semigroup $\{T(t)\}_{t \geq 0}$ on $X$ with $D(A)$ representing the domain of the linear operator (usually unbounded) $A, F$ is an upper-Carathéodory multifunction, and the nonlocal term $g$ is a given function from $C(J, X)$ into $X$.

Using the technique of Hausdorff measure of noncompactness and the multivalued fixed point theorem, we prove some existence results on the fractional semilinear nonlocal differential inclusion (1.1)-(1.2). We assume that the semigroup $\{T(t)\}_{t \geq 0}$ is equicontinuous. Note that the case of compact $\{T(t)\}_{t \geq 0}$ is just a particular case of our assumption. Therefore, the results in the present paper extend to some extent those in $[23,26]$.

This paper is organized as follows. In Section 2, we present some relevant definitions and facts about fractional derivative and integral, the Hausdorff measure of noncompactness, and the set-valued analysis. In Section 3, we give the existence results of mild solutions for problem (1.1)-(1.2). In Section 4, an example is given to briefly show a potential application of our results.

\section{Preliminaries}

Let $\mathbb{N}, \mathbb{R}$, and $\mathbb{R}^{+}$be the sets of positive integers, real numbers, and positive real numbers, respectively. Also, we define the following sets of subsets of $X$ :

$P(X)=\{B \subseteq X: B$ is nonempty and bounded $\}$,

$P_{c l}(X)=\{B \subseteq X: B$ is nonempty and closed $\}$,

$P_{c p}(X)=\{B \subseteq X: B$ is nonempty and compact $\}$,

$P_{c l, c v}(X)=\{B \subseteq X: B$ is nonempty, closed, and convex $\}$, and

$P_{c p, c v}(X)=\{B \subseteq X: B$ is nonempty, compact, and convex $\}$.

Now let us recall the definitions of fractional derivative and integral. 
Definition 1 The Riemann-Liouville fractional integral of order $q>0$ with the lower limit zero for a function $f$ is defined as

$$
I^{q} f(t)=\frac{1}{\Gamma(q)} \int_{0}^{t}(t-s)^{q-1} f(s) \mathrm{d} s, \quad t>0,
$$

provided that the right-hand side of $(2.1)$ is pointwise defined on $[0, \infty)$, where $\Gamma$ is the gamma function.

Definition 2 The Caputo fractional derivative of order $q>0$ with the lower limit zero for a function $f$ is defined as

$$
{ }^{C} D_{t}^{q} f(t)=\frac{1}{\Gamma(n-q)} \int_{0}^{t}(t-s)^{n-q-1} f^{(n)}(s) \mathrm{d} s, \quad t>0,
$$

where $n \in \mathbb{N}$ is such that $q \in(n-1, n)$.

If $f$ is an abstract function with values in $X$, then the integrals in the Definitions 1 and 2 are taken in the Bochner sense. In particular, when $0<q<1$, we have

$$
{ }^{C} D_{t}^{q} f(t)=\frac{1}{\Gamma(1-q)} \int_{0}^{t} \frac{f^{\prime}(s)}{(t-s)^{q}} \mathrm{~d} s, \quad t>0
$$

Now using the probability density function and its Laplace transform developed in [27], we give the following definition of mild solutions to problem (1.1)-(1.2).

Definition 3 A function $u \in C(J, X)$ is said to be a mild solution of (1.1)-(1.2) if $u(0)=g(u)$ and there exists $f \in L^{1}(J, X)$ such that $f(t) \in F(t, u(t))$ and

$$
u(t)=\mathcal{T}_{q}(t) g(u)+\int_{0}^{t}(t-s)^{q-1} \mathcal{S}_{q}(t-s) f(s) \mathrm{d} s
$$

for $t \in J$, where

$$
\begin{aligned}
& \mathcal{T}_{q}(t)=\int_{0}^{\infty} h_{q}(\theta) T\left(t^{q} \theta\right) \mathrm{d} \theta, \\
& \mathcal{S}_{q}(t)=q \int_{0}^{\infty} \theta h_{q}(\theta) T\left(t^{q} \theta\right) \mathrm{d} \theta .
\end{aligned}
$$

Here $h_{q}$ is the probability density function on $(0, \infty)$ given by

$$
h_{q}(\theta)=\frac{1}{q} \theta^{-1-1 / q} \bar{\omega}_{q}\left(\theta^{-1 / q}\right) \text {, }
$$

where

$$
\bar{\omega}_{q}(\theta)=\frac{1}{\pi} \sum_{n=1}^{\infty}(-1)^{n-1} \theta^{-n q-1} \frac{\Gamma(n q+1)}{n !} \sin (\pi n q), \quad \theta \in(0, \infty) .
$$


Note that $h_{q}(\theta) \geq 0$ for $\theta \in(0, \infty)$ and it satisfies

$$
\begin{aligned}
& \int_{0}^{\infty} h_{q}(\theta) \mathrm{d} \theta=1 \\
& \int_{0}^{\infty} \theta^{v} h_{q}(\theta) \mathrm{d} \theta=\frac{\Gamma(1+v)}{\Gamma(1+q v)}, \quad v \in[0,1] .
\end{aligned}
$$

It should be pointed out that Definition 3 can be regarded as a generalization of many previous definitions of mild solutions for various differential equations [22-26, 28-31], in particular, the definitions used by Zhou and Jiao [22], Li et al. [23], and Ji and Li [24] for the following nonlocal fractional semilinear differential equation:

$$
\begin{aligned}
& { }^{C} D_{t}^{q} u(t)=A u(t)+f(t, u(t)), \quad t \in J, \\
& u(0)=g(u),
\end{aligned}
$$

and the definition introduced by Wang and Zhou [26] for the following local fractional semilinear differential inclusion:

$$
\begin{aligned}
& { }^{C} D_{t}^{q} u(t) \in A u(t)+F(t, u(t)), \quad t \in J, \\
& u(0)=u_{0} .
\end{aligned}
$$

Lemma 1 The linear operators $\mathcal{T}_{q}(t)$ and $\mathcal{S}_{q}(t)$ have the following properties:

(1) For any fixed $t \geq 0$, both $\mathcal{T}_{q}(t)$ and $\mathcal{S}_{q}(t)$ are bounded operators, that is, for any $x \in X$, we have [24]

$$
\begin{aligned}
\left\|\mathcal{T}_{q}(t) x\right\| & \leq M\|x\|, \\
\left\|\mathcal{S}_{q}(t) x\right\| & \leq \frac{M q}{\Gamma(1+q)}\|x\|,
\end{aligned}
$$

where $M=\sup _{t \in J}\|T(t)\|$.

(2) Both $\mathcal{T}_{q}(t)$ and $\mathcal{S}_{q}(t)$ are equicontinuous for $t \in J$ if $\{T(t)\}_{t \geq 0}$ is equicontinuous.

Proof Property (1) follows directly from the definitions of $\mathcal{T}_{q}(t)$ and $\mathcal{S}_{q}(t)$, the definition of the constant $M$, and properties (2.8) and (2.9) of the probability density function. To show property (2), we shall show that both $\left\{\mathcal{T}_{q}(t) x: x \in B\right\}$ and $\left\{\mathcal{S}_{q}(t) x: x \in B\right\}$ are equicontinuous for any bounded subset $B$ of $X$. To this end, let $0 \leq t<t+h \leq b$, and let $\varepsilon>0$ be small enough. We then have

$$
\begin{aligned}
\left\|\mathcal{T}_{q}(t+h) x-\mathcal{T}_{q}(t) x\right\|= & \left\|\int_{0}^{\infty} h_{q}(\theta) T\left((t+h)^{q} \theta\right) x \mathrm{~d} \theta-\int_{0}^{\infty} h_{q}(\theta) T\left(t^{q} \theta\right) x \mathrm{~d} \theta\right\| \\
\leq & \int_{0}^{\infty} h_{q}(\theta)\left\|T\left((t+h)^{q} \theta\right) x-T\left(t^{q} \theta\right) x\right\| \mathrm{d} \theta \\
\leq & \int_{0}^{\varepsilon} h_{q}(\theta)\left\|T\left((t+h)^{q} \theta\right) x-T\left(t^{q} \theta\right) x\right\| \mathrm{d} \theta \\
& +\int_{\varepsilon}^{\infty} h_{q}(\theta)\left\|T\left((t+h)^{q} \theta\right) x-T\left(t^{q} \theta\right) x\right\| \mathrm{d} \theta
\end{aligned}
$$




$$
\begin{aligned}
\leq & 2 M\|x\| \int_{0}^{\varepsilon} h_{q}(\theta) \mathrm{d} \theta \\
& +\int_{\varepsilon}^{\infty} h_{q}(\theta)\left\|T\left((t+h)^{q} \theta\right) x-T\left(t^{q} \theta\right) x\right\| \mathrm{d} \theta .
\end{aligned}
$$

By using (2.8) and the absolute continuity of the integral it is easy to see that the term $2 M\|x\| \int_{0}^{\varepsilon} h_{q}(\theta) \mathrm{d} \theta$ can be made arbitrarily small by choosing $\varepsilon$ sufficiently small. On the other hand, it follows from the Lebesgue dominated convergence theorem and the equicontinuity of $T(t)$ for $t>0$ that, as $h \rightarrow 0$, we have

$$
\int_{\varepsilon}^{\infty} h_{q}(\theta)\left\|T\left((t+h)^{q} \theta\right) x-T\left(t^{q} \theta\right) x\right\| \mathrm{d} \theta \rightarrow 0 .
$$

Therefore, $\left\|\mathcal{T}_{q}(t+h) x-\mathcal{T}_{q}(t) x\right\| \rightarrow 0$ as $h \rightarrow 0$. So $\mathcal{T}_{q}(t)$ is equicontinuous.

In the same way we can prove that $\left\|\mathcal{S}_{q}(t+h) x-\mathcal{S}_{q}(t) x\right\| \rightarrow 0$ as $h \rightarrow 0$, uniformly for all $x \in B$, and thus $\mathcal{S}_{q}(t)$ is also equicontinuous.

We recall that for a bounded subset $B$ of the Banach space $X$, its Hausdorff measure of noncompactness is defined as

$$
\beta(B)=\inf \{\varepsilon>0: B \text { has a finite } \varepsilon \text {-net in } X\} .
$$

Some basic properties of the Hausdorff measure are presented in the following lemmas.

Lemma 2 ([32]) Let $X$ be a real Banach space. Let $B \subseteq X$ and $C \subseteq X$ be bounded. Then

(1) $B$ is relatively compact if and only if $\beta(B)=0$;

(2) $\beta(B)=\beta(\bar{B})=\beta(\operatorname{conv} B)$, where $\bar{B}$ and conv $B$ represent the closure and the convex hull of $B$, respectively;

(3) $\beta(B) \leq \beta(C)$ when $B \subseteq C$;

(4) $\beta(B+C) \leq \beta(B)+\beta(C)$, where $B+C=\{x+y: x \in B, y \in C\}$;

(5) $\beta(B \cup C) \leq \max \{\beta(B), \beta(C)\}$;

(6) $\beta(\lambda(B)) \leq|\lambda| \beta(B)$ for any $\lambda \in \mathbb{R}$;

(7) If the map $Q: D(Q) \subseteq X \rightarrow Z$ is Lipschitz continuous with constant $k$, then $\beta_{Z}(Q B) \leq k \beta(B)$ for any bounded subset $B \subseteq D(Q)$, where $Z$ is a Banach space. and $\beta_{Z}(\cdot)$ is the Hausdorff measure of noncompactness associated with $Z$;

(8) If $\left\{W_{n}\right\}_{n=1}^{\infty}$ is a decreasing sequence of bounded and closed nonempty subsets of $X$ and $\lim _{n \rightarrow \infty} \beta\left(W_{n}\right)=0$, then $\bigcap_{n=1}^{\infty} W_{n}$ is nonempty and compact in $X$.

Lemma 3 ([32]) If $W \subseteq C(J, X)$ is bounded and equicontinuous, then $\beta(W(t))$ is continuous on $J$, and

$$
\beta(W)=\sup _{t \in J} \beta(W(t))
$$

Lemma 4 ([33]) If $\left\{u_{n}\right\}_{n=1}^{\infty} \subset L^{1}(J, X)$ is uniformly integrable, then $\beta\left(\left\{u_{n}(t)\right\}_{n=1}^{\infty}\right)$ is measurable, and

$$
\beta\left(\left\{\int_{0}^{t} u_{n}(s) \mathrm{d} s\right\}_{n=1}^{\infty}\right) \leq 2 \int_{0}^{t} \beta\left(\left\{u_{n}(s)\right\}_{n=1}^{\infty}\right) \mathrm{d} s .
$$


Lemma 5 ([34]) If $B \subseteq X$ is bounded, then for each $\varepsilon>0$, there is a sequence $\left\{u_{n}\right\}_{n=1}^{\infty}$ in $B$ such that

$$
\beta(B) \leq 2 \beta\left(\left\{u_{n}\right\}_{n=1}^{\infty}\right)+\varepsilon
$$

In addition, for completeness, we further include some basic definitions and results on multivalued maps. For more details about the multivalued maps, see the books by Deimling [35] and $\mathrm{Hu}$ and Papageorgiou [36].

Definition 4 Let $X$ and $Y$ be two topological spaces.

(1) A multivalued map $F: X \rightarrow P(Y)$ is said to be convex (closed) valued if $F(x)$ is convex (closed) in $Y$ for all $x \in X$. Recall that $P(Y)$ represents the set of all nonempty and bounded subsets of $Y$.

(2) $F$ is said to be completely continuous if $F(B)$ is relatively compact for every bounded subset $B$ of $X$.

(3) $F$ is said to have a fixed point if there is $x \in X$ such that $x \in F(x)$.

(4) $F$ is said to be upper semicontinuous (u.s.c.) on $X$ if $F^{-1}(V)=\{x \in X: F(x) \subseteq V\}$ is an open subset of $X$ for every open subset $V$ of $Y$.

(5) $F$ is said to be closed if its graph $G_{F}=\{(x, y) \in X \times Y: y \in F(x)\}$ is a closed subset of the topological space $X \times Y$, that is, $x_{n} \rightarrow x, y_{n} \rightarrow y$, and $y_{n} \in F\left(x_{n}\right)$ imply $y \in F(x)$.

Remark 1 Note that if $D \subset X$ is closed, $F(x)$ is closed for all $x \in D$, and $\overline{F(D)}$ is compact, then $F$ is u.s.c. if and only if $F$ is closed.

Definition 5 A sequence $\left\{f_{n}\right\}_{n=1}^{\infty} \subset L^{1}(J, X)$ is said to be semicompact if

(1) It is integrably bounded, that is, there is $\omega \in L^{1}\left(J, \mathbb{R}^{+}\right)$such that

$$
\left\|f_{n}(t)\right\| \leq \omega(t) \quad \text { for a.e. } t \in J .
$$

(2) The set $\left\{f_{n}(t): n \in \mathbb{N}\right\}$ is relatively compact in $X$ for a.e. $t \in J$.

Lemma 6 ([37]) Every semicompact sequence in $L^{1}(J, X)$ is weakly compact in the space $L^{1}(J, X)$.

Lemma 7 ([26]) For $\theta \in(0,1]$ and $0<a \leq b$, we have

$$
\left|a^{\theta}-b^{\theta}\right| \leq(b-a)^{\theta} .
$$

\section{Main results}

Using the Hausdorff measure of noncompactness and the multivalued fixed point theorem, we shall give existence results for the nonlocal initial value problem (1.1)-(1.2). First, we list the hypotheses of our first main result.

(HA) The $C_{0}$ semigroup $\{T(t)\}_{t \geq 0}$ generated by the linear operator $A$ is equicontinuous. We denote

$$
M=\sup _{t \in J}\{\|T(t)\|\}
$$


(Hg) The nonlocal term $g: C(J, X) \rightarrow X$ is continuous and compact, and there exists a constant $N>0$ such that $\|g(u)\| \leq N$ for all $u \in C(J, X)$.

(HF) The multivalued operator $F: J \times X \rightarrow P_{c p, c v}(X)$ satisfies the following hypotheses:

(1) $F$ is measurable to $t$ for every $x \in X$ and u.s.c. to $x$ for a.e. $t \in J$. For every $u \in C(J, X)$, the set

$$
S_{F}(u)=\left\{f \in L^{1}(J, X): f(t) \in F(t, u(t)) \text {, a.e. } t \in J\right\}
$$

is nonempty.

(2) There exists a function $m \in L^{1 / q_{1}}\left(J, \mathbb{R}^{+}\right)$with $q_{1} \in(0, q)$ such that, for any $x \in X$,

$$
\|F(t, x)\|=\sup \{\|y\|: y \in F(t, x)\} \leq m(t)
$$

for a.e. $t \in J$.

(3) There exists a constant $L>0$ such that, for any bounded subset $D$ of $X$, we have

$$
\beta(F(t, D)) \leq L \beta(D)
$$

for a.e. $t \in J$.

$(\mathrm{H} \eta)$ There exists a function $\eta \in C\left(J, \mathbb{R}^{+}\right)$such that, for each constant $\lambda \in(-1,0)$, we have

$$
\eta(t) \geq M N+\frac{q M}{\Gamma(1+q)} \frac{t^{(1+\lambda)\left(1-q_{1}\right)}}{(1+\lambda)^{\left(1-q_{1}\right)}}\|m\|_{L^{1 / q_{1}[0, t]}}, \quad t \in J
$$

where $M, N, q_{1}$, and $m$ are from (HA), (Hg), and (HF)(2).

In the proof of the existence results, we also need the following lemmas.

Lemma 8 ([38]) Let $X$ be a Banach space, and $F$ be a multivalued mapping satisfying $(\mathrm{HF})(1)$. Let $\mathcal{F}: L^{1}(J, X) \rightarrow C(J, X)$ be a linear continuous mapping. Then the operator

$$
\mathcal{F} \circ S_{F}: C(J, X) \rightarrow P_{c l, c v}(C(J, X))
$$

where $\left(\mathcal{F} \circ S_{F}\right)(u)=\mathcal{F}\left(S_{F}(u)\right)$, is a closed graph operator in $C(J, X) \times C(J, X)$.

Lemma 9 ([39] (Fixed point theorem)) If $W$ is a bounded, closed, convex, and compact nonempty subset of $X$ and the map $F: W \rightarrow 2^{W}$ is upper semicontinuous with $F(x)$ being a closed and convex nonempty subset of $W$ for each $x \in W$, then $F$ has at least one fixed point in $W$.

Lemma 10 ([40]) Suppose that $b \geq 0, \sigma>0$, and $a(t)$ is a nonnegative function locally integrable on $0 \leq t<b$, and suppose that $c(t)$ is nonnegative and locally integrable on $0 \leq$ $t<b$ with

$$
c(t) \leq a(t)+b \int_{0}^{t}(t-s)^{\sigma-1} c(s) \mathrm{d} s, \quad t \in[0, b) .
$$


Then

$$
c(t) \leq a(t)+\mu \int_{0}^{t} E_{\sigma}^{\prime}(\mu(t-s)) a(s) \mathrm{d} s, \quad t \in[0, b),
$$

where

$$
\mu=(b \Gamma(\sigma))^{1 / \sigma}, \quad E_{\sigma}(z)=\sum_{n=0}^{\infty} \frac{z^{n \sigma}}{\Gamma(n \sigma+1)}, \quad E_{\sigma}^{\prime}(z)=\frac{d}{d z} E_{\sigma}(z) .
$$

Now we are ready to prove the following existence result for the nonlocal fractional differential inclusion (1.1)-(1.2).

Theorem 1 If hypotheses (HA), (Hg), (HF)(1)(2)(3), and (H $\eta)$ are satisfied, then the fractional differential inclusion (1.1)-(1.2) has at least one mild solution on $J$.

Proof In view of (HF)(1), for each $u \in C(J, X)$, the set $S_{F}(u)$ is nonempty. So we can define the operator $R: C(J, X) \rightarrow 2^{C(, X)}$ by

$$
R(u)=\left\{v \in C(J, X): v(t)=\mathcal{T}_{q}(t) g(u)+\int_{0}^{t}(t-s)^{q-1} \mathcal{S}_{q}(t-s) f(s) \mathrm{d} s, f \in S_{F}(u)\right\} .
$$

Then, obviously, the fixed point of the operator $R$ is a mild solution of (1.1)-(1.2). So we shall show that $R$ satisfies all the conditions of Lemma 9. For convenience, we subdivide the proof into four steps.

Step 1. We show that the values of $R$ are convex and closed subsets in $C(J, X)$.

We first prove that $R(u)$ is convex for arbitrary $u \in C(J, X)$. Indeed, if $v_{1}$ and $v_{2}$ belong to $R(u)$, then there exist $f_{1}$ and $f_{2}$ belonging to $S_{F}(u)$ such that, for every $t \in J$ and $i=1,2$,

$$
v_{i}(t)=\mathcal{T}_{q}(t) g(u)+\int_{0}^{t}(t-s)^{q-1} \mathcal{S}_{q}(t-s) f_{i}(s) \mathrm{d} s .
$$

Let $\lambda \in[0,1]$. Then, for every $t \in J$ and $i=1,2$, we have

$$
\lambda v_{1}(t)+(1-\lambda) v_{2}(t)=\mathcal{T}_{q}(t) g(u)+\int_{0}^{t}(t-s)^{q-1} \mathcal{S}_{q}(t-s)\left(\lambda f_{1}(s)+(1-\lambda) f_{2}(s)\right) \mathrm{d} s .
$$

It is easy to see that $S_{F}(u)$ is convex since $F$ has convex values. So $\lambda f_{1}+(1-\lambda) f_{2} \in S_{F}(u)$, and thus $\lambda v_{1}+(1-\lambda) v_{2} \in R(u)$. Therefore, $R(u)$ is convex.

We now prove that $R(u)$ is closed for every $u \in C(J, X)$. To this end, suppose that $\left\{v_{n}\right\}_{n=1}^{\infty}$ is a sequence in $R(u)$ such that $v_{n} \rightarrow v$ as $n \rightarrow \infty$. Then we need to show that $v \in R(u)$. According to the definition of $R$, there exists a sequence $\left\{f_{n}\right\}_{n=1}^{\infty} \subset S_{F}(u)$ such that, for every $t \in J$, we have

$$
v_{n}(t)=\mathcal{T}_{q}(t) g(u)+\int_{0}^{t}(t-s)^{q-1} \mathcal{S}_{q}(t-s) f_{n}(s) \mathrm{d} s .
$$

For any $n \geq 1$ and almost all $s \in(0, t]$, using assumption (HF)(2), we obtain

$$
\left\|f_{n}(s)\right\| \leq m(s)
$$


This shows that $\left\{f_{n}: n \geq 1\right\}$ is integrably bounded. Moreover, $\left\{f_{n}(t): n \geq 1\right\} \subset F(t, u(t))$ implies that $\left\{f_{n}(t): n \geq 1\right\}$ is relatively compact in $X$ for a.e. $t \in J$. Therefore, the set $\left\{f_{n}\right.$ : $n \geq 1\}$ is semicompact. By Lemma 6 it is weakly compact in $L^{1}(J, X)$. We can assume that $f_{n}$ converges weakly to a function $f \in L^{1}(J, X)$. Then by Mazur's lemma there is a sequence $\left\{g_{n}\right\}_{n=1}^{\infty} \subseteq \overline{\operatorname{conv}}\left\{f_{n}: n \geq 1\right\}$ such that $g_{n}$ converges strongly to $f$. Since the values of $F$ are convex and compact, we have that the set $S_{F}(u)$ is convex and compact. So $\left\{g_{n}\right\}_{n=1}^{\infty} \subseteq S_{F}(u)$, and $f \in S_{F}(u)$.

Moreover, for all $t \in J, s \in(0, t]$ and $n \geq 1$, we have

$$
\left\|(t-s)^{q-1} \mathcal{S}_{q}(t-s) f_{n}(s)\right\| \leq(t-s)^{q-1} \frac{M q}{\Gamma(1+q)} m(s)
$$

Let $\lambda=(q-1) /\left(1-q_{1}\right) \in(-1,0)$. Using Hölder's inequality, we get

$$
\begin{aligned}
\int_{0}^{t}(t-s)^{q-1} m(s) \mathrm{d} s & \leq\left(\int_{0}^{t}(t-s)^{\frac{q-1}{1-q_{1}}} \mathrm{~d} s\right)^{1-q_{1}}\left(\int_{0}^{t}(m(s))^{\frac{1}{q_{1}}} \mathrm{~d} s\right)^{q_{1}} \\
& \leq \frac{b^{(1+\lambda)\left(1-q_{1}\right)}}{(1+\lambda)^{\left(1-q_{1}\right)}}\|m\|_{L^{1 / q_{1}[0, b]}}<+\infty
\end{aligned}
$$

Therefore, according to the Lebesgue dominated convergence theorem, taking $n \rightarrow \infty$ on both sides of (3.4), we get

$$
v(t)=\mathcal{T}_{q}(t) g(u)+\int_{0}^{t}(t-s)^{q-1} \mathcal{S}_{q}(t-s) f(s) \mathrm{d} s
$$

which means that $v \in R(u)$.

Step 2. We construct a bounded, convex, closed, and compact nonempty subset $W \subseteq$ $C(J, X)$ such that $R$ maps $W$ into itself.

Let $W_{0}=\{u \in C(J, X):\|u(t)\| \leq \eta(t), t \in J\}$. It is obvious that $W_{0} \subseteq C(J, X)$ is bounded, closed, and convex. We claim that $R\left(W_{0}\right) \subseteq W_{0}$. To prove this, let $u \in W_{0}$ and $v \in R(u)$. Then there exists $f \in S_{F}(u)$ such that

$$
v(t)=\mathcal{T}_{q}(t) g(u)+\int_{0}^{t}(t-s)^{q-1} \mathcal{S}_{q}(t-s) f(s) \mathrm{d} s
$$

According to (2.10) and assumptions (HF)(2) and (H $\eta)$, for every $t \in J$, we have

$$
\begin{aligned}
\|v(t)\| & \leq\left\|\mathcal{T}_{q}(t) g(u)\right\|+\left\|\int_{0}^{t}(t-s)^{q-1} \mathcal{S}_{q}(t-s) f(s) \mathrm{d} s\right\| \\
& \leq M N+\frac{q M}{\Gamma(1+q)} \int_{0}^{t}(t-s)^{q-1} m(s) \mathrm{d} s \\
& \leq M N+\frac{q M}{\Gamma(1+q)} \frac{t^{(1+\lambda)\left(1-q_{1}\right)}}{(1+\lambda)^{\left(1-q_{1}\right)}}\|m\|_{L^{1 / q_{1}[0, t]}} \\
& \leq \eta(t)
\end{aligned}
$$

which implies that $R\left(W_{0}\right) \subseteq W_{0}$. 
Moreover, we claim that $R\left(W_{0}\right)$ is equicontinuous on $J$. For $0 \leq t<t+h \leq b$ and any $v \in R\left(W_{0}\right)$, there exist $u \in W_{0}$ and $f \in S_{F}(u)$ such that

$$
\begin{aligned}
\|v(t+h)-v(t)\| \leq & \left\|\mathcal{T}_{q}(t+h) g(u)-\mathcal{T}_{q}(t) g(u)\right\|+\| \int_{0}^{t+h}(t+h-s)^{q-1} \mathcal{S}_{q}(t+h-s) f(s) \mathrm{d} s \\
& -\int_{0}^{t}(t-s)^{q-1} \mathcal{S}_{q}(t-s) f(s) \mathrm{d} s \| \\
\leq & G_{1}+G_{2}+G_{3}+G_{4},
\end{aligned}
$$

where

$$
\begin{aligned}
& G_{1}=\left\|\mathcal{T}_{q}(t+h) g(u)-\mathcal{T}_{q}(t) g(u)\right\|, \\
& G_{2}=\left\|\int_{0}^{t}\left[(t+h-s)^{q-1}-(t-s)^{q-1}\right] \mathcal{S}_{q}(t+h-s) f(s) \mathrm{d} s\right\|, \\
& G_{3}=\left\|\int_{0}^{t}(t-s)^{q-1}\left[\mathcal{S}_{q}(t+h-s)-\mathcal{S}_{q}(t-s)\right] f(s) \mathrm{d} s\right\|, \\
& G_{4}=\left\|\int_{t}^{t+h}(t+h-s)^{q-1} \mathcal{S}_{q}(t+h-s) f(s) \mathrm{d} s\right\| .
\end{aligned}
$$

We now show that $G_{i} \rightarrow 0$ as $h \rightarrow 0$ for $i=1,2,3,4$. First, by assumption (HA) and Lemma 1(2) we know that $\left\{\mathcal{T}_{q}(t): t \in J\right\}$ is equicontinuous. Combining this with the compactness of $g$, we have that $G_{1} \rightarrow 0$ as $h \rightarrow 0$, uniformly for all $u \in W_{0}$.

Next, for $G_{3}$, from the equicontinuity of $\left\{\mathcal{S}_{q}(t): t \in J\right\}$, we can conclude that

$$
\int_{0}^{t}\left\|(t-s)^{q-1}\left[\mathcal{S}_{q}(t+h-s)-\mathcal{S}_{q}(t-s)\right] f(s)\right\| \mathrm{d} s \rightarrow 0
$$

as $h \rightarrow 0$, uniformly for all $f \in S_{F}(u)$ and $u \in W_{0}$. That is, $G_{3} \rightarrow 0$ as $h \rightarrow 0$.

Then for $G_{2}$, we note that $\lambda=(q-1) /\left(1-q_{1}\right) \in(-1,0)$. So for $s<t \leq t+h$, we have $(t-$ $s)^{\lambda} \geq(t+h-s)^{\lambda}$. Applying Lemma 7 and noting that $1-q_{1} \in(0,1)$, we get

$$
\left|\left[(t+h-s)^{\lambda}\right]^{1-q_{1}}-\left[(t-s)^{\lambda}\right]^{1-q_{1}}\right| \leq\left[(t-s)^{\lambda}-(t+h-s)^{\lambda}\right]^{1-q_{1}}
$$

Accordingly, we have

$$
\begin{aligned}
\left|(t+h-s)^{q-1}-(t-s)^{q-1}\right| & =\left|\left[(t+h-s)^{\lambda}\right]^{1-q_{1}}-\left[(t-s)^{\lambda}\right]^{1-q_{1}}\right| \\
& \leq\left[(t-s)^{\lambda}-(t+h-s)^{\lambda}\right]^{1-q_{1}}
\end{aligned}
$$

Therefore, by (3.1), Lemma 1(1), and Lemma 7, we get

$$
\begin{aligned}
G_{2} & \leq \int_{0}^{t}\left|(t+h-s)^{q-1}-(t-s)^{q-1}\right|\left\|\mathcal{S}_{q}(t+h-s) f(s)\right\| \mathrm{d} s \\
& \leq \frac{M q}{\Gamma(1+q)} \int_{0}^{t}\left|(t+h-s)^{q-1}-(t-s)^{q-1}\right| m(s) \mathrm{d} s \\
& \leq \frac{M q}{\Gamma(1+q)}\left(\int_{0}^{t}\left|(t+h-s)^{q-1}-(t-s)^{q-1}\right| \frac{1}{1-q_{1}} \mathrm{~d} s\right)^{1-q_{1}}\|m\|_{L^{1 / q_{1}}[0, b]}
\end{aligned}
$$




$$
\begin{aligned}
& \leq \frac{M q}{\Gamma(1+q)}\left(\int_{0}^{t}\left[(t-s)^{\lambda}-(t+h-s)^{\lambda}\right] \mathrm{d} s\right)^{1-q_{1}}\|m\|_{L^{1 / q_{1}[0, b]}} \\
& \leq \frac{M q}{(1+\lambda)^{\left(1-q_{1}\right)} \Gamma(1+q)}\left[h^{1+\lambda}+t^{1+\lambda}-(t+h)^{1+\lambda}\right]^{1-q_{1}}\|m\|_{L^{1 / q_{1}[0, b]}} \\
& \leq \frac{M q}{(1+\lambda)^{\left(1-q_{1}\right)} \Gamma(1+q)} h^{(1+\lambda)\left(1-q_{1}\right)}\|m\|_{L^{1 / q_{1}[0, b]}} .
\end{aligned}
$$

Therefore, $G_{2} \rightarrow 0$ as $h \rightarrow 0$.

Finally, for $G_{4}$, we have

$$
\begin{aligned}
G_{4} & \leq \int_{t}^{t+h}\left|(t+h-s)^{q-1}\right|\left\|\mathcal{S}_{q}(t+h-s) f(s)\right\| \mathrm{d} s \\
& \leq \frac{M q}{\Gamma(1+q)} \int_{t}^{t+h}(t+h-s)^{q-1} m(s) \mathrm{d} s \\
& \leq \frac{M q}{\Gamma(1+q)}\left(\int_{t}^{t+h}\left[(t+h-s)^{q-1}\right]^{\frac{1}{1-q_{1}}} \mathrm{~d} s\right)^{1-q_{1}}\|m\|_{L^{1 / q_{1}[t, t+h]}} \\
& \leq \frac{M q}{\Gamma(1+q)(1+\lambda)^{\left(1-q_{1}\right)}} h^{(1+\lambda)\left(1-q_{1}\right)}\|m\|_{L^{1 / q_{1}[t, t+h]}} .
\end{aligned}
$$

So we also have that $G_{4} \rightarrow 0$ as $h \rightarrow 0$. Therefore, we get $\|v(t+h)-v(t)\| \rightarrow 0$ as $h \rightarrow 0$, implying that $R\left(W_{0}\right)$ is equicontinuous on $J$.

Now, let us define a sequence $\left\{W_{n}\right\}_{n=1}^{\infty}$ recursively by $W_{n}=\overline{\operatorname{conv}} R\left(W_{n-1}\right)$ for all $n \geq 1$. From the above discussions we know that $W_{n} \subseteq W_{n-1}$ for all $n \geq 1$. Thus, $\left\{W_{n}\right\}_{n=1}^{\infty}$ is a decreasing sequence of closed, bounded, convex, and equicontinuous subsets of $C(J, X)$. Set $W=\bigcap_{n=1}^{\infty} W_{n}$. Then $W$ is a closed, bounded, convex, and equicontinuous subset of $C(J, X)$ and $R(W) \subseteq W$. We claim that $W$ is nonempty and compact in $C(J, X)$. Indeed, by Lemma 2 and (2.15), for arbitrary $\varepsilon>0$, there exist sequences $\left\{u_{k}\right\}_{k=1}^{\infty} \subset W_{n}$ and $\left\{f_{k}\right\}_{k=1}^{\infty} \subset$ $S_{F}\left(u_{k}\right)$ such that

$$
\begin{aligned}
\beta\left(W_{n+1}(t)\right) & =\beta\left(\overline{\operatorname{conv}}\left(R W_{n}\right)(t)\right)=\beta\left(\left(R W_{n}\right)(t)\right) \\
& \leq 2 \beta\left(\left\{R u_{k}(t)\right\}_{k=1}^{\infty}\right)+\varepsilon \\
& \leq 2 \beta\left(\mathcal{T}_{q}(t) g\left(\left\{u_{k}\right\}_{k=1}^{\infty}\right)+\int_{0}^{t}(t-s)^{q-1} \mathcal{S}_{q}(t-s)\left\{f_{k}(s)\right\}_{k=1}^{\infty} \mathrm{d} s\right)+\varepsilon \\
& \leq 2 \beta\left(\mathcal{T}_{q}(t) g\left(\left\{u_{k}\right\}_{k=1}^{\infty}\right)\right)+2 \beta\left(\int_{0}^{t}(t-s)^{q-1} \mathcal{S}_{q}(t-s)\left\{f_{k}(s)\right\}_{k=1}^{\infty} \mathrm{d} s\right)+\varepsilon
\end{aligned}
$$

Since $g$ is compact, by Lemma 2, (2.14), and (3.2) we have

$$
\begin{aligned}
\beta\left(W_{n+1}(t)\right) & \leq 4 \int_{0}^{t}(t-s)^{q-1} \beta\left(\mathcal{S}_{q}(t-s)\left\{f_{k}(s)\right\}_{k=1}^{\infty}\right) \mathrm{d} s+\varepsilon \\
& \leq \frac{4 q M}{\Gamma(1+q)} \int_{0}^{t}(t-s)^{q-1} \beta\left(F\left(s, W_{n}(s)\right)\right) \mathrm{d} s+\varepsilon \\
& \leq \frac{4 q M L}{\Gamma(1+q)} \int_{0}^{t}(t-s)^{q-1} \beta\left(W_{n}(s)\right) \mathrm{d} s+\varepsilon
\end{aligned}
$$


Since (3.6) is true for arbitrary $\varepsilon>0$, we must have

$$
\beta\left(W_{n+1}(t)\right) \leq \frac{4 q M L}{\Gamma(1+q)} \int_{0}^{t}(t-s)^{q-1} \beta\left(W_{n}(s)\right) \mathrm{d} s
$$

Because $\left\{W_{n}\right\}_{n=1}^{\infty}$ is decreasing with respect to $n$, we define

$$
\mu(t)=\lim _{n \rightarrow \infty} \beta\left(W_{n}(t)\right), \quad t \in J .
$$

Taking $n \rightarrow \infty$ on both sides of (3.7), we have

$$
\mu(t) \leq \frac{4 q M L}{\Gamma(1+q)} \int_{0}^{t}(t-s)^{q-1} \mu(s) \mathrm{d} s
$$

By Lemma 10 we have that $\mu(t)=0, t \in J$. On the other hand, $\left\{W_{n}\right\}_{n=1}^{\infty}$ is bounded and equicontinuous. Hence, by Lemma 3 we get $\beta\left(W_{n}\right)=\sup _{t \in J} \beta\left(W_{n}(t)\right)$, which implies that $\lim _{n \rightarrow \infty} \beta\left(W_{n}\right)=0$. Then it follows from Lemma 2(8) that $W=\bigcap_{n=1}^{\infty} W_{n}$ is nonempty and compact in $C(J, X)$.

Step 3. We show that the graph of $R$ is closed.

Let $\left\{u_{n}\right\}_{n=1}^{\infty} \subset W$ with $u_{n} \rightarrow u$ as $n \rightarrow \infty, v_{n} \in R\left(u_{n}\right)$, and $v_{n} \rightarrow v$ as $n \rightarrow \infty$. We shall show that $v \in R(u)$. Since $v_{n} \in R\left(u_{n}\right)$, there exists $f_{n} \in S_{F}\left(u_{n}\right)$ such that

$$
v_{n}(t)=\mathcal{T}_{q}(t) g\left(u_{n}\right)+\int_{0}^{t}(t-s)^{q-1} \mathcal{S}_{q}(t-s) f_{n}(s) \mathrm{d} s .
$$

In the sequel, we must show that there exists $f \in S_{F}(u)$ such that

$$
v(t)=\mathcal{T}_{q}(t) g(u)+\int_{0}^{t}(t-s)^{q-1} \mathcal{S}_{q}(t-s) f(s) \mathrm{d} s .
$$

Consider the linear operator $\mathcal{F}: L^{1}(J, X) \rightarrow C(J, X)$ defined as

$$
\mathcal{F}(f)(t)=\int_{0}^{t}(t-s)^{q-1} \mathcal{S}_{q}(t-s) f(s) \mathrm{d} s
$$

Clearly, $\mathcal{F}$ is linear and continuous. Hence, it follows from Lemma 8 that $\mathcal{F} \circ S_{F}$ is a closed graph operator. Moreover, we have

$$
v_{n}(\cdot)-\mathcal{T}_{q}(\cdot) g\left(u_{n}\right) \in \mathcal{F}\left(S_{F}\left(u_{n}\right)\right)
$$

Since $v_{n} \rightarrow v, u_{n} \rightarrow u$, and $g$ is continuous, we obtain

$$
v(\cdot)-\mathcal{T}_{q}(\cdot) g(u) \in \mathcal{F}\left(S_{F}(u)\right)
$$

that is,

$$
v(t)-\mathcal{T}_{q}(t) g(u)=\int_{0}^{t}(t-s)^{q-1} \mathcal{S}(t-s) f(s) \mathrm{d} s
$$

for some $f \in S_{F}(u)$, and thus the graph of $R$ is closed. 
Step 4 . We show that $R$ is u.s.c. on $W$.

As a consequence of the previous proof, we have that $W$ is closed and $R(u)$ is closed for all $u \in W$. The set $\overline{R(W)} \subseteq W$ is compact. Moreover, $R$ is closed. According to Remark 1 , we can come to the conclusion that $R$ is u.s.c.

Finally, due to the fixed point of Lemma $9, R$ has at least one point $u \in R(u)$, and $u$ is a mild solution to the fractional semilinear differential inclusion (1.1) with the nonlocal condition (1.2).

Remark 2 If $A$ generates an analytic semigroup or a differential semigroup $\{T(t)\}_{t \geq 0}$, then $\{T(t)\}_{t \geq 0}$ is equicontinuous [41]. In applications of partial differential equations, such as parabolic equations and strongly damped wave equations, the corresponding solution semigroups are analytic. Therefore, the results in this paper have wide applicability.

In Theorem 1, hypothesis (HF)(2) may be sometimes difficult to be verified, and also the mapping $g$ needs to be uniformly bounded. Indeed, if $g$ is compact, then $g$ is bounded on a bounded subset. Next, we further give an existence result for the fractional differential inclusion (1.1)-(1.2) under a relatively weak condition of $F$ when $g$ is not uniformly bounded. For this purpose, we introduce the following two hypotheses:

$\left(\mathrm{Hg}^{\prime}\right) \quad$ The nonlocal term $g: C(J, X) \rightarrow X$ is continuous and compact.

(HF)(2') There exist a function $\alpha \in L^{1 / q_{1}}\left(J, \mathbb{R}^{+}\right)$for some given $q_{1} \in(0, q)$ and an increasing function $\Omega: \mathbb{R}^{+} \rightarrow \mathbb{R}^{+}$such that

$$
\|F(t, x)\| \leq \alpha(t) \Omega(\|x\|)
$$

for a.e. $t \in J$ and all $x \in X$.

Theorem 2 Assume that hypotheses (HA), $\left(\mathrm{Hg}^{\prime}\right)$, and (HF)(1)(2')(3) are satisfied. Suppose that

$$
\lim _{k \rightarrow \infty} \sup \left\{\frac{M}{k}\left(\gamma(k)+\frac{M_{1} q}{(1+\lambda)^{\left(1-q_{1}\right)} \Gamma(1+q)} \Omega(k) b^{(1+\lambda)\left(1-q_{1}\right)}\right)\right\}<1,
$$

where $\gamma(k)=\sup \{\|g(u)\|:\|u\| \leq k\}, M_{1}=\|\alpha\|_{L^{1 / q_{1}}[0, b]}$, and $\lambda=(q-1) /\left(1-q_{1}\right) \in(-1,0)$. Then the fractional differential inclusion (1.1)-(1.2) has at least one mild solution on $J$.

Proof By (3.8) there exists a constant $k>0$ such that

$$
M\left(\gamma(k)+\frac{M_{1} q}{(1+\lambda)^{\left(1-q_{1}\right)} \Gamma(1+q)} \Omega(k) b^{(1+\lambda)\left(1-q_{1}\right)}\right)<k .
$$

Let $W_{0}=\{u \in C(J, X):\|u\| \leq k\}$ and $W_{1}=\overline{\operatorname{conv}} R\left(W_{0}\right)$. Then for any $v \in W_{1}$, there exist $u \in W_{0}$ and $f \in S_{F}(u)$ such that

$$
v(t)=\mathcal{T}_{q}(t) g(u)+\int_{0}^{t}(t-s)^{q-1} \mathcal{S}_{q}(t-s) f(s) \mathrm{d} s .
$$

From (2.10) and assumption $\left(\mathrm{Hg}^{\prime}\right)$ we conclude that, for every $t \in J$, we have 


$$
\begin{aligned}
\|v(t)\| & \leq\left\|\mathcal{T}_{q}(t) g(u)\right\|+\left\|\int_{0}^{t}(t-s)^{q-1} \mathcal{S}_{q}(t-s) f(s) \mathrm{d} s\right\| \\
& \leq M\left(\gamma(k)+\frac{q}{\Gamma(1+q)} \Omega(k) \int_{0}^{t}(t-s)^{q-1} \alpha(s) \mathrm{d} s\right) \\
& \leq M\left(\gamma(k)+\frac{q}{\Gamma(1+q)} \Omega(k)\left(\int_{0}^{t}(t-s)^{\frac{q-1}{1-q_{1}}} \mathrm{~d} s\right)^{\left(1-q_{1}\right)}\|\alpha\|_{L^{1 / q_{1}[0, b]}}\right) \\
& \leq M\left(\gamma(k)+\frac{M_{1} q}{(1+\lambda)^{\left(1-q_{1}\right)} \Gamma(1+q)} \Omega(k) b^{(1+\lambda)\left(1-q_{1}\right)}\right) \\
& <k .
\end{aligned}
$$

It follows that $\|v\|=\sup _{t \in J}\{\|v(t)\|\}<k$. Then $W_{1} \subseteq W_{0}$. Set $W_{n}=\overline{\operatorname{conv}} R\left(W_{n-1}\right)$ for all $n \geq 1$. Then we can complete the proof similarly to that of Theorem 1.

\section{An example}

We consider the following fractional partial differential inclusion:

$$
\left\{\begin{array}{l}
\partial_{t}^{q} x(t, y) \in \partial_{y}^{2} x(t, y)+u(t, x(t, y)) \\
x(t, 0)=x(t, \pi)=0 \\
x(0, y)=\int_{0}^{1} h(s) \sin (1+|x(s, y)|) \mathrm{d} s
\end{array}\right.
$$

where $X=L^{2}([0, \pi] ; \mathbb{R}), \partial_{t}^{q}$ is the Caputo fractional partial derivative of order $q$ with $0<$ $q<1, h \in L^{1}([0,1] ; \mathbb{R})$, and $u:[0,1] \times X \rightarrow P(X)$.

We define the operator $A$ by $A z=z^{\prime \prime}$ with $D(A)=\left\{z \in X: z\right.$ and $z^{\prime}$ are absolutely continuous, $\left.z^{\prime \prime} \in X, z(0)=z(\pi)=0\right\}$. Then

$$
A z=-\sum_{n=1}^{\infty} n^{2}\left\langle z, e_{n}\right\rangle e_{n}, \quad z \in D(A)
$$

where $e_{n}(z)=(1 / \pi)^{1 / 2} \sin n z, 0 \leq z \leq \pi, n=1,2, \ldots$. Clearly, $A$ generates a strongly continuous semigroup $\{T(t)\}_{t \geq 0}$ in $X$, which is compact and analytic, and it is given by

$$
T(t) z=\sum_{n=1}^{\infty} e^{-n^{2} t}\left\langle z, e_{n}\right\rangle e_{n}, \quad z \in X .
$$

This implies that $A$ satisfies assumption (HA).

Then system (4.1) can be reformulated as

$$
\left\{\begin{array}{l}
{ }^{C} D_{t}^{q} x(t) \in A x(t)+F(t, x(t)), \\
x(0)=g(x),
\end{array}\right.
$$

where $x(t)(y)=x(t, y), t \in[0,1], y \in[0, \pi]$, and $F(t, x(t))(y)=u(t, x(t, y))$. The function $g$ : $C([0,1], X) \rightarrow X$ is given by

$$
g(x)(y)=\int_{0}^{1} h(s) \sin (1+|x(s, y)|) \mathrm{d} s .
$$


If we impose suitable conditions on the above-defined functions for verifying the assumptions in Theorem 1 or Theorem 2, then we can conclude that (4.1) admits at least one mild solution on $[0,1]$.

\section{Competing interests}

The authors declare that they have no competing interests.

\section{Authors' contributions}

All authors contributed equally to the writing of this paper. All authors read and approved the final manuscript.

\section{Author details}

'School of Mathematics and Physics, Yancheng Institute of Technology, Yancheng, Jiangsu 224051, P.R. China.

${ }^{2}$ Department of Mathematics and Statistics, University of North Carolina at Charlotte, Charlotte, NC 28223, United States.

\section{Acknowledgements}

The authors thank the support of the National Natural Science Foundation of China (Grant No. 11471281) for the work reported in this paper.

Received: 18 October 2016 Accepted: 28 November 2016 Published online: 08 December 2016

\section{References}

1. Rossikhin, YA, Shitikova, MV: Application of fractional derivatives to the analysis of damped vibrations of viscoelastic single mass systems. Acta Mech. 120, 109-125 (1997)

2. Poinot, T, Trigeassou, JC: Identification of fractional systems using an output-error technique. Nonlinear Dyn. 38 133-154 (2004)

3. Tan, W, Pan, W, Xu, M: A note on unsteady flows of a viscoelastic fluid with the fractional Maxwell model between two parallel plates. Int. J. Non-Linear Mech. 38, 645-650 (2003)

4. Tan, $\mathrm{W}, \mathrm{Xu}, \mathrm{M}$ : Unsteady flows of a generalized second grade fluid with the fractional derivative model between two parallel plates. Acta Mech. Sin. 20, 471-476 (2004)

5. Tan, W, Fu, C, Fu, C, Xie, W, Cheng, H: An anomalous subdiffusion model for calcium spark in cardiac myocytes. Appl. Phys. Lett. 91, 183901 (2007)

6. Xue, C, Nie, J, Tan, W: An exact solution of start-up flow for the fractional generalized Burgers' fluid in a porous half-space. Nonlinear Anal. TMA 69, 2086-2094 (2008)

7. Xue, C, Nie, J: Exact solutions of the Rayleigh-Stokes problem for a heated generalized second grade fluid in a porous half-space. Appl. Math. Model. 33, 524-531 (2009)

8. Wang, J, Zhou, Y: A class of fractional evolution equations and optimal controls. Nonlinear Anal., Real World Appl. 12, 262-272 (2011)

9. Podlubny, I: Fractional Differential Equations. Academic Press, San Diego (1999)

10. Sabatier, J, Agrawal, OP, Tenreiro Machado, JA: Advances in Fractional Calculus: Theoretical Developments and Applications in Physics and Engineering. Springer, Dordrecht (2007)

11. Kilbas, AA, Srivastava, HM, Trujillo, JJ: Theory and Applications of Fractional Differential Equations. Elsevier, Amsterdam (2006)

12. Eidelman, SD, Kochubei, AN: Cauchy problem for fractional diffusion equations. J. Differ. Equ. 199, $211-255$ (2004)

13. Lakshmikantham, V, Vatsala, AS: Basic theory of fractional differential equations. Nonlinear Anal. TMA 69, 2677-2682 (2008)

14. Agarwal, RP, Lakshmikantham, V, Nieto, JJ: On the concept of solution for fractional differential equations with uncertainty. Nonlinear Anal. TMA 72, 2859-2862 (2010)

15. Byszewski, L, Lakshmikantham, V: Theorem about the existence and uniqueness of a solution of a nonlocal abstract Cauchy problem in a Banach space. Appl. Anal. 40, 11-19 (1991)

16. Byszewski, L: Theorems about the existence and uniqueness of solutions of a semilinear evolution nonlocal Cauchy problem. J. Math. Anal. Appl. 162, 494-505 (1991)

17. Ntouyas, SN, Tsamatos, PC: Global existence for semilinear evolution equations with nonlocal conditions. J. Math. Anal. Appl. 210, 679-687 (1997)

18. Fan, Z, Li, G: Existence results for semilinear differential equations with nonlocal and impulsive conditions. J. Funct. Anal. 258, 1709-1727 (2010)

19. Aizicovici, S, Staicu, V: Multivalued evolution equations with nonlocal initial conditions in Banach spaces. Nonlinear Differ. Equ. Appl. 14, 361-376 (2007)

20. Liang, J, Liu, JH, Xiao, T: Nonlocal impulsive problems for nonlinear differential equations in Banach spaces. Math. Comput. Model. 49, 798-804 (2009)

21. Zhu, L, Li, G: Nonlocal differential equations with multivalued perturbations in Banach spaces. Nonlinear Anal. 69 2843-2850 (2008)

22. Zhou, Y, Jiao, F: Nonlocal Cauchy problem for fractional evolution equations. Nonlinear Anal., Real World Appl. 11 4465-4475 (2010)

23. Li, K, Peng, J, Gao, J: Nonlocal fractional semilinear differential equations in separable Banach spaces. Electron. J. Differ. Equ. 2013, 7 (2013)

24. Ji, S, Li, G: Solutions to nonlocal fractional differential equations using a noncompact semigroup. Electron. J. Differ. Equ. 2013, 240 (2013)

25. Ji, S: Approximate controllability of semilinear nonlocal fractional differential systems via an approximating method. Appl. Math. Comput. 236, 43-53 (2014) 
26. Wang, J, Zhou, Y: Existence and controllability results for fractional semilinear differential inclusions. Nonlinear Anal., Real World Appl. 12, 3642-3653 (2011)

27. El-Borai, MM: Some probability densities and fundamental solutions of fractional evolution equations. Chaos Solitons Fractals 14, 433-440 (2002)

28. Zhou, Y, Jiao, F: Existence of mild solutions for fractional neutral evolution equations. Comput. Math. Appl. 59, 1063-1077 (2010)

29. Liu, X, Liu, Z, Fu, X: Relaxation in nonconvex optimal control problems described by fractional differential equations. J. Math. Anal. Appl. 409, 446-458 (2014)

30. Chadha, A, Pandey, DN: Existence and approximation of solution to neutral fractional differential equation with nonlocal conditions. Comput. Math. Appl. 69, 893-908 (2015)

31. Ge, F, Zhou, H, Kou, C: Approximate controllability of semilinear evolution equations of fractional order with nonlocal and impulsive conditions via an approximating technique. Appl. Math. Comput. 275, 107-120 (2016)

32. Banas, J, Goebel, K: Measure of Noncompactness in Banach Spaces. Lect. Notes Pure Appl. Math., vol. 60. Dekker, New York (1980)

33. Heinz, HP: On the behavior of measures of noncompactness with respect to differentiation and integration of vector-valued functions. Nonlinear Anal. TMA 7, 1351-1371 (1983)

34. Bothe, D: Multivalued perturbations of $m$-accretive differential inclusions. Isr. J. Math. 108, 109-138 (1998)

35. Deimling, K: Multivalued Differential Equations. de Gruyter, Berlin (1992)

36. Hu, S, Papageorgiou, NS: Handbook of Multivalued Analysis. Kluwer Academic, Dordrecht (2000)

37. Kamenskii, M, Obukhovskii, V, Zecca, P: Condensing Multivalued Maps and Semilinear Differential Inclusions in Banach Spaces. De Gruyter Series in Nonlinear Analysis and Applications, vol. 7. de Gruyter, Berlin (2001)

38. Lasota, A, Opial, Z: An application of the Kakutani-Ky Fan theorem in the theory of ordinary differential equations. Bull. Acad. Pol. Sci., Sér. Sci. Math. Astron. Phys. 13, 781-786 (1965)

39. Agarwal, RP, Meehan, M, O'regan, D: Fixed Point Theory and Applications. Cambridge Tracts in Mathematics. Cambridge University Press, Cambridge (2001)

40. Henry, D: Geometric Theory of Semilinear Parabolic Equations. Lecture Notes in Math., vol. 840. Springer, New York (1981)

41. Pazy, A: Semigroups of Linear Operators and Applications to Partial Differential Equations. Springer, New York (1983)

\section{Submit your manuscript to a SpringerOpen ${ }^{\circ}$ journal and benefit from:}

- Convenient online submission

Rigorous peer review

- Immediate publication on acceptance

- Open access: articles freely available online

- High visibility within the field

- Retaining the copyright to your article 